\title{
Open-door policies
}

\begin{abstract}
Nature research journals announce new reporting summaries to promote transparency, and our editors welcome early-career researchers to the Springer Nature office in New York to discuss careers in scientific publishing.
\end{abstract}

\section{$\mathbf{S}$}

Toward greater reproducibility ince 2013, Nature and the Nature research journals have asked authors of papers in the life sciences to complete a checklist when they submit a paper. This extra step-prompting authors to disclose important elements of experimental design and analysiswas part of a broader effort to improve the quality of reporting in our life sciences articles.

We now go further. Alongside every life sciences article, we will publish a new reporting summary document, to which authors are expected to add details of experimental design, reagents and analysis. This is another step in encouraging transparency, ensuring that papers contain sufficient methodological detail, and improving statistics reviewing and reporting.

We expect that the new reporting summary will assist reviewers and editors in assessing experimental quality and help readers to locate crucial details on data collection and analysis. Those familiar with the original checklist will find similar elements in the new reporting summary. The summary also has a strong focus on points that are known to be sources of experimental variability and that tend to be poorly reported in the literature. When appropriate, the new general reporting summary will be accompanied by more specific and more detailed accessory reporting summaries for papers based on certain techniques, such as ChIP-seq.

Nature has long been interested in promoting the reproducibility of published results. Although the issues that give rise to the 'reproducibility crisis' are multifaceted and come into play long before a paper is submitted, our responsibility is to ensure that the research we publish is well planned, well executed and well reported.

As with the initial checklist, these documents aim to improve reporting, rather than to enforce a defined set of standards. They should make apparent the details of how a study was designed, performed and analyzed, to allow reviewers and readers to interpret the results and understand any limitations. There are, of course, separate experimental standards that must be met to comply with our editorial policies, and these are captured in our new editorial policy checklist.

As a complement to these new documents, we will now mandate greater transparency in data presentation. We will ask authors, where possible, not to use bar graphs and instead to use approaches that present full data distribution. We have also expanded our data-deposition mandates to include proteomics data, and our policy on reporting of cell line authentication is being extended to all papers with data generated using cell lines. We have also added a reporting table for cryo-EM, which joins those for atomic structures from NMR and X-ray crystallography.

With these and other steps, we will continue to work to ensure the rigor of the work we publish and to promote the ability of the community to build on this research.

\section{An invitation from the Nature journal editors}

As editors for Nature and Nature research journals, most of our communications with authors and reviewers are focused on the work we publish. However, on some occasions, the focus is reversed, and we are asked to describe the work we do. Most often, we are queried informally about our jobs at conference poster sessions, breaks and meals, but we also regularly speak to researchers about our work at the request of meeting organizers or during site visits to universities and research institutions. We welcome these opportunities both to 'lift the veil' on the editorial process and to share our work experience with those who may be considering an editorial career. The expanded facilities in our new location at 1 New York Plaza now enable us to host early-career researchers at our New York offices as well.

We have recently started organizing visits of up to 25 graduate students and postdocs from local institutions. These $\sim 1.5$-hour gatherings typically take place over lunch and are designed to maximize opportunities for participants to ask questions about careers in scientific publishing and the editorial process. Editors from different journals and at different stages of their careers-Associate, Senior, Chief and Executive Editors-each provide a brief description of their scientific background, what attracted them to scientific editing at Nature Research, and how the rewards and responsibilities change with experience and career progression. Human Resources representatives are also present to provide information on job search and application policies.

Questions from the audience cover a broad range of topics: what our typical day is like (How many manuscripts do we handle? How many do we send out for review? How often do we travel for conferences?), queries about editorial tasks (How do we find reviewers? How do reviewer comments inform our decisions? How do we handle appeals?), what we look for in editors (Do you need postdoctoral experience? What is the interview process like? How do you prepare for a career in editing?), and the interface between expectation and experience (What do you like most and least about your job? What surprised you the most when you moved from the bench to the editor's desk?). These meetings provide an opportunity for each of us to convey the most personally and professionally fulfilling aspects of our work that we hope inspires similarly minded individuals to join our editorial teams.

Such in-person meetings were influential in our own decisions to become Nature journal editors, and it has been gratifying to see the positive response to these events so far. Our New York outreach events are intended to complement the editorial recruitment program at Springer Nature (www.springernature.com/editorial-and-publishingjobs). If you are interested in visiting us in New York, please contact nsmb@us.nature.com. 